Main Outcome Measures Independent risk factors associated with all MEs (both injury- and illness-related) using race day factors and individual self-reported medical history (allergies and medical conditions/medication use) were investigated using a multivariate model with a Poisson distribution, reporting the prevalence ratio (PR: 95\% CI).

Results Over 5 years 1749 medical encounters were recorded. Independent risk factors associated with all MEs were: older females (males 31-40years vs females $>50$ years, 0.6: 0.4-0.8, $\mathrm{p}=0.0017$; males $41-50$ years vs females $>50$ years, $0.5: 0.4-$ $0.7, \mathrm{p}=0.0002$; males $>50$ years vs females $>50$ years, 0.6 : $0.4-0.8, \mathrm{p}=0.0008)$, slower race pace $(7-<8 \mathrm{~min} / \mathrm{km}$ vs $8-<9$ $\mathrm{min} / \mathrm{km}, 0.8: 0.7-0.9 ; \mathrm{p}=0.0044)$ and self-reported medical conditions/medication use (no history vs. medical condition/ medication use, 0.7: 0.6-0.9; $\mathrm{p}=0.0082$ ).

Conclusion A self-reported history of a medical condition/medication, older females, and slower runners were risk factors associated with MEs during/immediately after the $90 \mathrm{~km}$ Comrades ultramarathon. These data support initiatives to introduce pre-race medical screening, which could then form the basis for the design and implementation of prevention programs to manage risk of MEs at these events.

\section{METABOLIC/ENDOCRINE DISEASE, OLDER FEMALES, LONGER RACE DISTANCE, SLOWER RACE PACE AND HIGHER WBGT ARE INDEPENDENT RISK FACTORS ASSOCIATED WITH MEDICAL ENCOUNTERS IN $21.1 \mathrm{KM}$ AND 56 KM RUNNERS: A SAFER STUDY IN 76654 STARTERS}

\begin{abstract}
1,2,3 Martin Schwellnus, ${ }^{1}$ Nicola Sewry, ${ }^{4,5,6}$ Mats Borjesson, ${ }^{7}$ Sonja Swanevelder, ${ }^{7,8}$ Esme Jordaan. 'Sport, Exercise Medicine and Lifestyle Institute (SEMLI), Pretoria, South Africa; ${ }^{2}$ IOC Research Centre, South Africa, Pretoria, South Africa; ${ }^{3}$ Emeritus Professor of Sport and Exercise Medicine, Faculty of Health Sciences, University of Cape Town, South Africa, Cape Town, South Africa; ${ }^{4}$ Institute of Neuroscience and Physiology, Sahlgrenska Academy, Göteborg University, Göteborg, Sweden; ${ }^{5}$ Center for Health and Performance, Göteborg University, Göteborg, Sweden; ${ }^{5}$ Sahlgrenska University Hospital/Östra, Göteborg, Göteborg, Sweden; ${ }^{7}$ Biostatistics Unit, South African Medical Research Council, Cape Town, South Africa; ${ }^{8}$ Statistics and Population Studies Department, University of the Western Cape, Cape Town, South Africa
\end{abstract}

\subsection{6/bjsports-2021-I0C.66}

Background Recent data indicate that pre-race medical screening and education can reduce medical encounters (MEs) at an endurance running event. However, the relationship between the risk of MEs and specific risk factors from pre-race medical screening, together with race day factors, and has not been explored.

Objective To determine the independent risk factors that are associated with MEs during distance running events, using data from pre-race medical screening and race day.

Design Prospective study, with cross-sectional analyses.

Setting 2012-2015 Two Oceans marathon races $(21.1 \mathrm{~km}$, $56 \mathrm{~km})$, South Africa.

Patients (or Participants) 76654 consenting race entrants.

Interventions (or Assessment of Risk Factors) All entrants completed a pre-race medical screening questionnaire upon entry, coupled with an educational intervention, as per their responses to questions. Race day data were collected from the race organisers and all MEs were recorded by medical staff on race day.

Main Outcome Measurements Risk factors associated with ME (both injury- and illness-related) were investigated using a multiple regression model with a Poisson distribution (reporting the prevalence ratio - PR: 95\% CI) that included: demographics (age, sex), race day data [wet-bulb globe temperature (WBGT), race distance $(21.1 \mathrm{~km}$ or $56 \mathrm{~km})$, race pace], and individual pre-race medical screening data.

Results Independent risk factors associated with MEs were: history of metabolic disease (2.1: $1.3-3.3 ; \mathrm{p}=0.0030)$, older females (>55years) $(2.5: 1.6-4.1 ; \mathrm{p}=0.0002)$, longer race distance $(56 \mathrm{~km}$ vs. $21.1 \mathrm{~km}, 1.9: 1.5-2.4 ; \mathrm{p}<0.0001)$, slower race pace (increase of $1 \mathrm{~min} / \mathrm{km}, 1.2: 1.1-1.3 ; \mathrm{p}=0.0029$ ), and higher WBGT $(\mathrm{p}=0.0264)$.

Conclusions Metabolic/endocrine disease, older females, longer race distance, slower race pace and higher WBGT were independent risk factors for MEs in distance running events. In addition to environmental factors, these data support initiatives to obtain pre-race medical screening, demographic, and running pace data in order to design and implement ME prevention programs at distance running events.

\section{SLOWER RUNNERS, OLDER FEMALES AND ROUTE CHARACTERISTICS ARE INDEPENDENT RISK FACTORS FOR SERIOUS/LIFE-THREATENING MEDICAL ENCOUNTERS IN A 90 KM ULTRAMARATHON: A SAFER STUDY IN 70328 RUNNERS OVER 5 YEARS}

1,2 Nicola Sewry, ${ }^{3}$ Jeremy Boulter, 1,2 Martin Schwellnus, ${ }^{1}$ Sport Exercise Medicine and Lifestyle Institute (SEMLI), Pretoria, South Africa; ${ }^{2}$ IOC Research Centre, South Africa, Pretoria, South Africa; ${ }^{3}$ Medical Director, Comrades Marathon, Pietermaritzburg, South Africa

\subsection{6/bjsports-2021-IOC.67}

Background Serious/life-threatening medical encounters (sltMEs) occur during mass community-based events, with higher incidences in ultra-endurance events. Risk factors associated with sltMEs are under-investigated.

Objective To determine independent risk factors for serious/ life-threatening MEs in a $90 \mathrm{~km}$ ultramarathon running event.

Design Retrospective clinical audit, cross-sectional analysis

Setting 2014-2018 Comrades ultramarathon $(90 \mathrm{~km}$, alternating annually between an 'up' vs. a 'down' run between a coastal and inland city), South Africa

\section{Participants 70328 race starters}

Interventions All entrants voluntarily completed two medicallyrelated questions: 1) history of allergies, and 2) history of any medical condition and/or medication use. Race day data (start time, finish time, age, sex, 'up' vs. 'down' run) was recorded by the race organisers; and sltMEs (as defined in the 2019 consensus statement on mass community-based events) were recorded by race medical staff during and immediately after the event.

Main Outcome Measures Independent risk factors associated with sltMEs (injury- and illness-related) using demographics (age, sex), race day factors (route 'up' or 'down'; race pace), and individual self-reported medical history (allergies, medical conditions/medication use) were investigated using a multivariate model with a Poisson distribution, reporting the prevalence ratio (PR: 95\% CI).

Results 159 serious/life-threatening medical encounters were recorded over the 5 years. The following independent risk factors were associated with serious/life-threatening medical encounters: age and sex (males $31-40$ yrs vs females $>50$ yrs, 0.3 : $0.1-0.7, p=0.0076$; males $41-50$ yrs vs females $>50$ yrs, 